\title{
A REFORMA DO SISTEMA DA ATENÇÃO HOSPITALAR BRASILEIRA: O QUÊ MUDA PARA O CAISM?
}

\author{
Mary Ângela Parpinelli ${ }^{1}$
}

\section{RESUMO}

A reforma do Sistema de Atenção Hospitalar tem como objetivo precípuo garantir a efetiva e plena inserção destes e, particularmente dos Hospitais de Ensino e Universitários (HU/HE), às necessidades do Sistema Único de Saúde (SUS). O HU/HE deve solicitar, junto ao Ministério da Saúde, a certificação e submeter-se a processo de avaliação. Uma vez certificado, o HU/HE passa a receber um fator de incentivo financeiro a partir de nova modalidade de contrato pelo SUS. O CAISM foi certificado e investirá o fator de incentivo financeiro na manutenção e atualização de seu parque tecnológico.

PALAVRAS-CHAVE: Reforma; Certificação de Hospitais de Ensino; Missão do CAISM.

\section{AS BASES PARA A REFORMA DA ATENÇÃO HOSPITALAR}

Impulsionado e fortalecido por um cenário de crise financeira nos hospitais. o Ministério da Saúde (MS) propõe a reforma do sistema de assistência hospitalar ( BRASIL, 2004), introduzindo nas discussões a mudança estrutural do modelo assistencial na saúde, da qual participa a assistência hospitalar.

O modelo vigente de assistência na saúde, a despeito da inserção do Programa de Saúde da Família (PSF), pelo MS em 1994, segundo os pensadores da reforma, permanece tecnoassistencial e hospitalocêntrico, ou seja, privilegia o procedimento, onde o compromisso do ato de assistir a saúde, em todos os níveis, faz-se por produção de consultas, exames e medicamentos e o hospital como o centro mais importante da atenção. O hospital deve deixar de ser a ponta do sistema para tornar-se um ponto em uma rede

\footnotetext{
${ }^{1}$ Docente do Departamento de Tocoginecologia da Faculdade de Ciências Médicas da UNICAMP e Diretora Executiva do CAISM.

\begin{tabular}{llllll}
\hline Serviço Social \& Saúde & Campinas & v. 4 & n. 4 & p. 1-156 & Maio 2005 \\
\hline
\end{tabular}
}


horizontal onde todos os serviços de atenção são importantes, contudo o centro de atuação e resolutividade estar representado pela atenção primária à saúde (CAMPOS, 1994).

A reorganização efetiva do modelo assistencial para sistemas integrados de serviços de saúde, com articulação e pactuação regional dinâmica, flexível e progressiva, como proposta, somente será atingida e sustentada a partir da incorporação de mudanças de conceito de atenção à saúde, revisões dos processos de trabalho e das relações entre os diferentes componentes do Sistema Único de Saúde (SUS). Entretanto, esta transformação é considerada essencial e necessária para garantir os objetivos do SUS: universalidade, integralidade e equidade.

Neste contexto, de forma sintetizada, a redefinição do papel do hospital, na reforma da atenção hospitalar brasileira conduzida pela Secretaria de Assistência à Saúde (SAS), MS, apóia-se em quatro eixos principais ou elementos de sustentação e inter-relação entre todas as ações estratégicas desenvolvidas:

Garantia de acesso com ampliação da cobertura das ações e, fundamentalmente, disponibilidade de atendimento acompanhado de contínua melhoria da qualidade dos serviços ofertados.

Humanização entendida não apenas, como um processo de melhoria das condições gerais de atendimento, mas também na ótica de abordagem e integração da gestão e da assistência à saúde, voltada às necessidades do usuário e seus familiares e não mais enfocada nas doenças e na conveniência dos profissionais de saúde.

Inserção na rede de assistência onde os gestores locais do SUS têm destacado papel na discussão das necessidades de saúde, da demanda de serviços e da efetiva condução e controle das ações implementadas pelo hospital. "Os hospitais não devem constituir-se em ilhas de excelência à parte da rede de serviços”.

Democratização da gestão onde os hospitais devem assumir o seu papel e responsabilidade social, trabalhando de forma transparente e ampliando os seus mecanismos de participação social.

A descentralização e a regionalização também têm sido consideradas elementos orientadores na construção de políticas de atenção hospitalar.

\begin{tabular}{llllll}
\hline Serviço Social \& Saúde & Campinas & v. 4 & n. 4 & p. 1-156 & Maio 2005
\end{tabular} 
Dadas as peculiaridades, a complexidade organizacional e de financiamento entre os hospitais universitários (HU) e de ensino (HE) no Brasil, para as proposições da reforma contou-se com um amplo estudo elaborado por uma Comissão Interinstitucional, criada a partir de Portaria Interministerial $n^{\circ}$ 562, de 12 de maio de 2003. É importante mostrar a abrangência e representatividade dos membros para a composição desta comissão: Conselho Nacional dos Secretários Estaduais de Saúde; Conselho Nacional dos Secretários Municipais de Saúde; Ministério da Saúde; Ministério da Educação; Ministério de Ciência e Tecnologia; Ministério de Planejamento, Orçamento e Gestão; Comissão de Educação e de Seguridade Social e Família da Câmara dos Deputados; Conselho Nacional de Saúde; Associação Brasileira dos Hospitais Universitários e de Ensino; Associação Nacional dos Dirigentes de Instituições Federais de Ensino Superior; Associação Brasileira de Reitores de Universidades Estaduais; Associação Brasileira de Educação Médica; Associação Brasileira de Enfermagem; Direção Nacional dos Estudantes de Medicina e Executiva Nacional dos Estudantes de Enfermagem.

A tarefa enfocou a responsabilidade dos $\mathrm{HU} / \mathrm{HE}$ para com os princípios e diretrizes do SUS e em suas proposições considerou a definição do novo modelo reservado aos HU - na saúde e na educação; sua relação com o SUS - inserção, regulação e integração; os desenhos organizacionais e de gestão - autonomia, eficiência e eficácia gerencial; o modelo de financiamento - global, contrato de gestão ou misto; a democratização e gestão participativa. Elaboraram-se os critérios para a re-certificação dos hospitais ora credenciados como de ensino (Portarias MEC/MS nº1.000; 1.005/2004). Criou-se o Programa de Reestruturação dos Hospitais de Ensino no SUS (Portarias GM/MS n 1.702 de 17 de agosto de 2004).

A etapa de certificação foi realizada mediante solicitação do hospital e cumpriu uma série de exigências documentais além da submissão de uma avaliação por representantes dos ministérios da educação e da saúde. Cumprida a etapa da certificação os hospitais efetivamente certificados como de ensino passaram a receber um fator de incentivo somado ao financiamento, a modalidade de contratação envolve a definição de metas, pactuadas com o gestor local do SUS, e uma nova modalidade de remuneração, atualmente mista, produção por teto nos procedimentos de alta complexidade e pela

\begin{tabular}{llllll}
\hline Serviço Social \& Saúde & Campinas & v. 4 & n. 4 & p. 1-156 & Maio 2005 \\
\hline
\end{tabular}


contratualização nas atividades de média complexidade, esta última contemplada através de indicadores com metas estabelecidas nos campos da atenção à saúde, do ensino e da formação, da pesquisa e da gestão, devendo ser acompanhadas e atestadas pelo conselho gestor do hospital e/ou pela comissão permanente de acompanhamento de contratos.

Atenção à Saúde através da produção de serviços de qualidade, adequados às necessidades de saúde da população de sua área de abrangência, sempre de forma pactuada com os gestores locais do SUS. O HU/HE deve ao planejar e programar suas atividades e ações considerar-se parte integrante da rede de serviços locorregionais de atenção integral à saúde. A orientação da atenção deve ser humanizada, centrada no usuário e seus familiares, selecionando e se aproximando das melhores e mais adequadas tecnologias na busca de resultados expressos pela melhoria nas condições de saúde e na qualidade de vida de seus usuários.

Educação e Desenvolvimento de Profissionais a partir do ensino de graduação, de pós-graduação, de profissionalização e de desenvolvimento de profissionais de saúde e para o sistema de saúde. A orientação do ensino deve atender aos princípios de excelência técnica, integralidade e humanização das práticas e pelas necessidades do sistema regional de saúde.

Produção de Conhecimento e Desenvolvimento de Tecnologia: as pesquisas realizadas nos HU/HE devem incluir temas clínicos e educacionais, coerentes com a proposta curricular dos cursos de graduação e de pós-graduação e com a realidade de saúde de cada região. Outro aspecto, neste mesmo objetivo, refere-se ao desenvolvimento e avaliação de novas tecnologias para o sistema de saúde e da sociedade à qual pertence.

Aprimoramento da gestão em três diretrizes: profissionalização da gestão administrativa buscando eficiência e eficácia; gestão hospitalar integrada com o SUS local e a incorporação das políticas nacionais do SUS.

\section{O CENTRO DE ATENÇÃO INTEGRAL À SAÚDE DA MULHER (CAISM)}

Conhecer a história, contada por seus ex-diretores, prévia a criação do CAISM auxiliará na compreensão sobre a sua inserção no complexo hospitalar da UNICAMP e no sistema de saúde.

\begin{tabular}{llllll}
\hline Serviço Social \& Saúde & Campinas & v. 4 & n. 4 & p. 1-156 & Maio 2005 \\
\hline
\end{tabular}


No início da década de 70, num período em que não havia cobertura pelo sistema público vigente, o Departamento de Tocoginecologia organizou o Programa de Controle de Câncer do Colo Uterino, posteriormente outros programas foram implantados como Programa de Câncer de Mama, Planejamento Familiar, Incentivo ao Aleitamento Materno e outros. Também foi criado o Ambulatório de Tocoginecologia Preventiva (PINOTTI, 2001; FAÚNDES, 2001; ZEFERINO, 2001).

Nesse período a Faculdade de Medicina tinha como hospital de ensino a Santa Casa de Misericórdia de Campinas e com o crescimento dos programas a demanda assistencial exigiu a elaboração de convênio, no caso com a Maternidade de Campinas, para a realização de cirurgias excedentes da Santa Casa, realizadas por profissionais da UNICAMP com a participação de médicos residentes do Departamento de Tocoginecologia, o convênio estendia-se aos atendimentos em Obstetrícia.

O pioneirismo na forma de abordar a questão da atenção integral à saúde da mulher e os bons resultados dos programas serviu de modelo para a implantação do Programa de Atenção Integral à Saúde da Mulher (PAISM), em nível nacional pelo Ministério da Saúde, em 1984.

Em 1985, a Faculdade de Medicina iniciava a transferência de suas atividades para o Hospital das Clínicas e o número de leitos destinados ao Departamento de Tocoginecologia seria basicamente o mesmo oferecido pela Santa Casa. Surgiu o projeto de um pequeno hospital com 35 leitos, denominado Centro de Câncer (CECAN) para atender às mulheres com câncer ginecológico. Apoiado na construção do CECAN e no PAISM surge o projeto de construção do CAISM (ZEFERINO, 2001).

O CAISM foi criado por portaria (GR 1001, de 15 de junho de 1984) e oficialmente iniciou suas atividades em 1986. Os objetivos estabelecidos em portaria foram: prestar assistência à Saúde da Mulher de forma integral e integrada; prestar assistência à saúde do recém-nascido de forma integral e integrada; realizar atividade de ensino e pesquisa dentro do campo da Atenção Integral à Saúde da Mulher e do Recémnascido.

Faúndes, 2001 destaca que no CAISM o modelo de atenção à mulher é integrado, onde ao cuidado em seus aspectos físicos (doença) realizado pelo médico e

\begin{tabular}{llllll}
\hline Serviço Social \& Saúde & Campinas & v. 4 & n. 4 & p. 1-156 & Maio 2005 \\
\hline
\end{tabular}


enfermeira somam-se os aspectos psicológicos e sociais realizados por psicólogas e assistentes sociais.

Ao longo de sua trajetória o CAISM vem modificando e adaptando seu perfil a luz das necessidades do sistema de saúde e das mudanças promovidas no ensino, principalmente no currículo médico.

Em 2003, acolhendo a solicitação da Universidade, o CAISM elaborou seu Planejamento Estratégico (PLANES), para este revisou sua missão, redefiniu seus princípios e valores e sua visão de futuro. O projeto foi amplamente discutido e a versão final aprovada pela Diretoria Executiva e pelo Conselho Técnico Administrativo. Após o projeto foi incorporado ao PLANES (CAISM/ UNICAMP, 2003) de toda a área de saúde da Faculdade de Ciências Médicas (FCM) e encaminhado a Comissão de Planejamento Estratégico Institucional (COPEI) no âmbito da reitoria.

A nova missão do CAISM apóia-se em quatro eixos: prestar assistência interprofissional e interdisciplinar integrada, especializada, regionalizada e referenciada no campo da saúde da mulher e do recém-nascido como centro de referência de maior complexidade no SUS; propiciar o desenvolvimento das atividades de ensino de graduação, pós-graduação e extensão em sintonia com as diretrizes da FCM; promover e propiciar atividades de formação, capacitação técnico-profissional e de educação continuada para os recursos humanos na área de saúde; promover e realizar atividades de pesquisa no campo da saúde da mulher e do recém-nascido.

Definiu-se para o cumprimento da missão os seguintes princípios: prestação de serviço de qualidade à sociedade em prol do bem estar coletivo, com inserção e responsabilidade social; respeito à dignidade da pessoa e aos seus direitos fundamentais; humanização nas relações com usuários, trabalhadores e alunos; conduta ética aos princípios da universidade e do SUS. Dentre os valores foram selecionados: ser público, de qualidade e com compromisso social; prestar atendimento humanizado, regionalizado e hierarquizado; ser parceiro nos três níveis de gerência do SUS: municipal, estadual e federal; promover equidade e a harmonia entre as áreas do CAISM; praticar gerência por processo de trabalho; praticar gestão participativa e transparente; incentivar e promover o

\begin{tabular}{llllll}
\hline Serviço Social \& Saúde & Campinas & v. 4 & n. 4 & p. 1-156 & Maio 2005 \\
\hline
\end{tabular}


reconhecimento e a valorização profissional; comprometer-se com a atualização e manutenção do parque tecnológico.

O CAISM definiu como visão de futuro consolidar-se como instituição pública e universitária de referência nacional e internacional, com excelência nas atividades de ensino, pesquisa, extensão e assistência de maior complexidade à saúde da mulher e do recém-nascido; integrada ao SUS.

Em meados de 2004 o CAISM em conjunto com o Hospital das Clínicas solicitou junto ao MS a certificação como hospital de ensino, sofreu avaliação no início do segundo semestre e ao final de 2004 foi re-certificado como hospital de ensino. O fator de incentivo financeiro, que passou a receber, será aplicado na manutenção e atualização do seu parque tecnológico.

\section{ALGUMAS PERSPECTIVAS SOBRE A REFORMA ${ }^{2}$}

A finalidade precípua da reforma é estabelecer a efetiva inserção dos hospitais, particularmente os HU/HE, às necessidades do SUS. Pressuposto estabelecido na constituição de 1988. Se obtiver êxito possibilitará a afirmação definitiva do SUS como um dos maiores e melhores sistemas de saúde pública e de assistência integral à saúde das pessoas.

A qualificação dos atendimentos se dará pela organização dos sistemas de referência e contra-referência com as demais unidades de saúde, pela humanização da assistência, pelas atividades de educação permanente dos profissionais dos hospitais e das redes municipais na construção de protocolos de cuidados clínicos.

A inserção dos HU/HE nas necessidades assistenciais do SUS terá também, a curto e médio prazo, efeito na qualidade de formação dos recursos humanos para o SUS, permitindo maior valorização e compreensão da lógica assistencial e ética do SUS e preparando-os melhor para o mercado de trabalho no qual as oportunidades de emprego na rede pública são crescentes e atrativas.

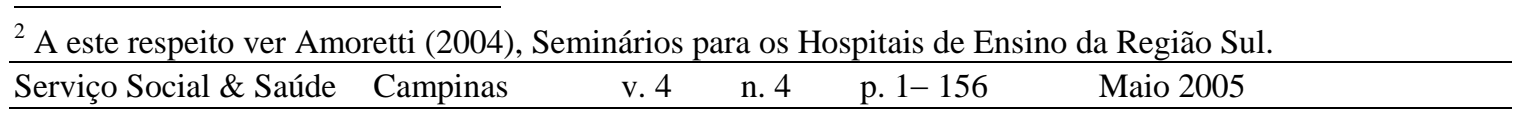


O financiamento, por contrato de gestão, mediante metas e indicadores (qualitativos e quantitativos), avalizado pelo gestor local possibilitará a qualificação da assistência e a correção das ofertas de serviços segundo as necessidades reais da população de cada região e estará re-valorizando o papel dos HU/HE frente a opinião pública, bem como corrigindo distorções históricas de financiamentos, favorecendo a sustentabilidade das instituições hospitalares.

\begin{abstract}
The most important aim of Hospital Care System reform is to ensure the effective and total insertion of this system into the Single Health Care System requirements (SUS), particularly the Teaching and University Hospitals (HU/HE). The HU/HE should request a certification from the Ministry of Health and undergo an evaluation process. Once certified, the HU/HE begins to receive a financial incentive factor arising from a new contract modality carried out by the SUS. CAISM was certified and will invest the financial incentive factor to maintain and upgrade its technological park.
\end{abstract}

KEY-WORDS: reform, certification of teaching hospitals, mission of CAISM.

\title{
REFERÊNCIAS BIBLIOGRÁFICAS
}

AMORETTI, R. Seminário para os Hospitais de Ensino da Região Sul. 2004 Promoção GHC; SMS/POA; ABRAHUE. BRASIL. http://www.ghc.com.br 2005

BRASIL. MINISTÉRIO DA SAÚDE. Reforma do Sistema da Atenção Hospitalar Brasileira. 2004 Brasília, DF.

CAISM/UNICAMP. Planejamento Estratégico (PLANES) 2003 Comunicação e Difusão do CAISM/DEC.

CAMPOS, G.W. A Saúde Pública e a Defesa da Vida. 1994: Hulcitec,São Paulo, SP

FAÚNDES, A. CAISM 15 anos, Consolidando sua História (entrevista). 2001 Comunicação e Difusão do CAISM/DEC.

\begin{tabular}{llllll}
\hline Serviço Social \& Saúde & Campinas & v. 4 & n. 4 & p. 1-156 & Maio 2005
\end{tabular} 
PINOTTI, J.A. CAISM 15 anos, Consolidando sua História (entrevista). 2001 Comunicação e Difusão do CAISM/DEC.

ZEFERINO, L.C. CAISM 15 anos, Consolidando sua História (entrevista). 2001 Comunicação e Difusão do CAISM/DEC. 
